\section{Binocular rivalry of equiluminant targets}

\author{
DAVID H. WESTENDORF and M. PAZ GALUPO \\ University of Arkansas, Fayetteville, Arkansas
}

\begin{abstract}
We describe a stimulus pattern designed for use in test probe investigations of binocular rivalry. The pattern consists of a contour-free disk that is identical in luminance in both eyes surrounded by a dimmer annulus in one eye and a brighter annulus in the other. Temporal characteristics of rival alternations can be controlled by manipulating luminance of the annuli. Sensitivity to probes inserted in the rival region can be measured uncontaminated by differences in the adaptation state of the eyes or pattern masking.
\end{abstract}

As part of his classic investigation of the effects of contrast on the perception of surface color, or perceived lightness, Wallach (Wallach \& Adams, 1954) found that monocular disks of nearly identical luminance could be made to engage in binocular rivalry if they were made to look different by virue of simultaneous contrast. The present paper describes a variant of Wallach's dichoptic stimulus arrangement that seems especially suitable for studies of binocular rivalry that employ test probes. Test probe studies of rivalry usually involve measuring the sensitivity of an eye during both its dominant and suppressed phases. In such experiments the state of the eye, dominant or suppressed, serves as an independent variable, and some measure of visual performance in responding to the probe stimulus is the dependant variable.

Many studies of binocular rivalry employ stimuli that are rivalrous by virtue of interocular differences in the retinal location or orientation of contours, a common version of which consists of orthogonally oriented cosine or squarewave grating patterns (see Figure 1A). Although such patterns are suitable for many purposes, their use in test probe experiments can introduce confounding effects. The problem is that not only is probe detection determined by the rivalry state of the detecting eye (dominant or suppressed), but it can also be influenced by pattern masking from the stimuli used to induce rivalry (Westendorf, 1989).

Stimuli with no contours in the rival region can be made rivalrous by virtue of being portrayed in reverse contrast. Figure 1B illustrates an example similar to one employed by Wales and Fox (1970) in a study of the effects of rivalry on increment flash detection. Although this stimulus con-

The authors are grateful to Randy Blake and T. J. Mueller for helpful comments concerning an earlier version of this manuscript. Correspondence should be addressed to $\mathrm{D}$. $\mathrm{H}$. Westendorf, Department of Psychology, University of Arkansas, Fayetteville, AR 72701. figuration obviates pattern masking problems, it carries other liabilities. For one thing, left and right eye performance cannot be compared unconfounded by the adaptation state of each retina. For another, only probes involving an increment in luminance can be inserted in the black disk.

Figure 1C illustrates the rival stimuli that we patterned after Wallach's. This reverse-contrast figure ground (RCFG) stimulus arrangement has none of the drawbacks associated with the stimuli shown in Figures $1 \mathrm{~A}$ and $1 \mathrm{~B}$. Each eye's stimulus consists of a disk surrounded by an annulus. The luminance of the disks is identical in each eye, whereas the luminance of the annuli is disparate. In one eye the annulus is dimmer than its disk, whereas in the other it is brighter. Viewed separately, the disks appear different in lightness because of simultaneous contrast. Superimposed binocularly, the disks engage in vigorous rivalry during which perception vacillates between that of the apparently lighter and apparently darker monocular disks. Except in the immediate vicinity of its inner border, the appearance of the fused annuli is quite stable and of a lightness intermediate between that of its monocular components.

Although the RCFG stimulus shown in Figure IC can be created in a variety of ways (e.g., photographically, on computer screens, etc.), in our laboratory they are electronically produced by generating rasters on the screens of four CRT monitors, two of which are mounted on each arm of a large mirror stereoscope and optically superimposed with beam splitters. High-contrast photographic film is affixed to the face of each monitor so that a centrally located circular region of the raster of one monitor on each arm is seen surrounded by an annulus produced by the raster of the optically superimposed second monitor on that arm. The luminance of the various monocular components making up this display is controlled by varying a dc voltage applied to the $z$-axis of the appropriate monitor. To help observers maintain proper eye alignment, each eye's disk-annulus display is flanked on either side by a series of vertically oriented light bars. These fusion bars are features of the photographic masks on the disk monitors and thus of the same luminance as the disks.

The monocular components of the RCFG stimulus appear different both by virtue of the apparent lightness of the disks and the reverse contrast of contours marking the disk-annulus borders. Apparently it is the border appearance that provides an unambiguous clue to observers as to which eye is suppressed at any moment during an ongoing episode of rivalry. This conclusion is based on our experience when we showed naive observers two versions of a dichoptic disk-annulus display and asked them to indicate, via a three-position switch, the exclusive visibil- 

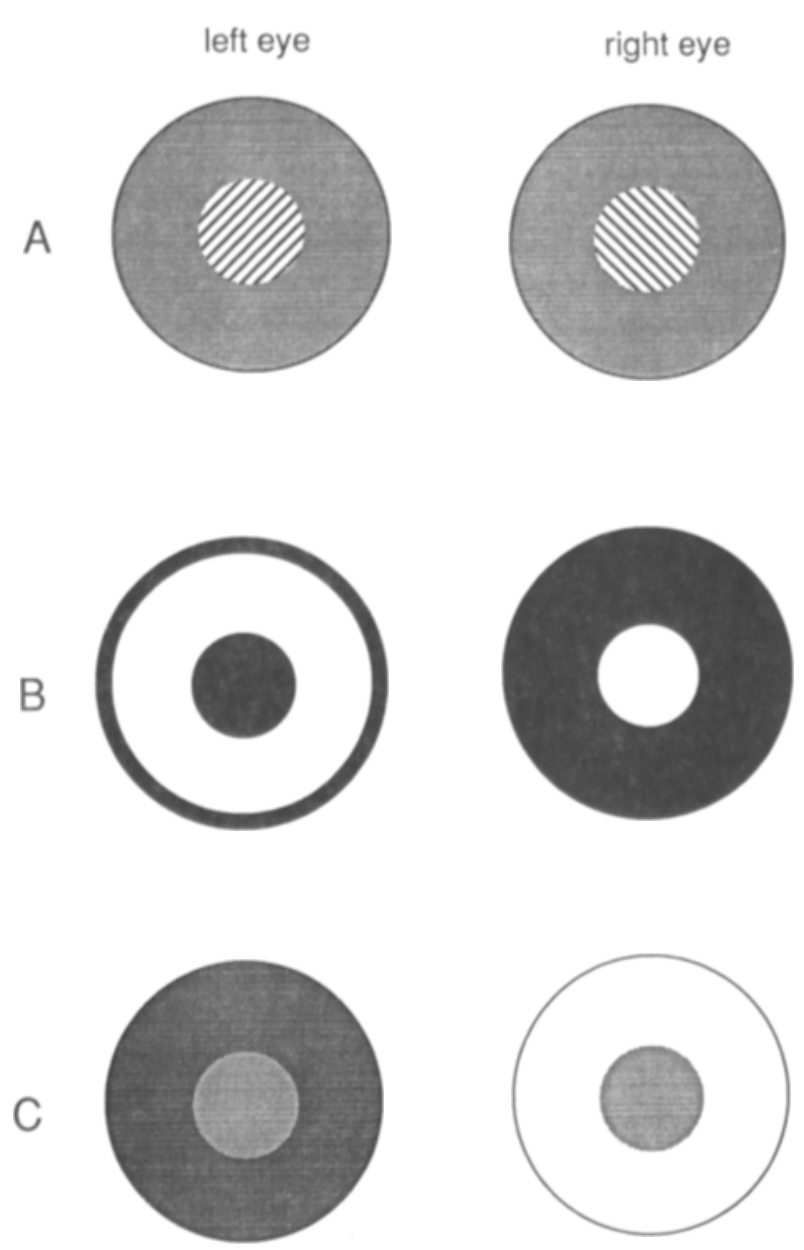

Figure 1. Three types of rival stimuli. (A) Orthogonal gratings. (B) Black-and-white reverse-contrast figure ground. (C) Reversecontrast figure ground with equiluminance.

ity of each monocular component of the display. (The center switch position was used to indicate periods of uncertain or mixed dominance.) The displays were located at a viewing distance so that each disk was $1^{\circ}$ in diameter and cotangent with the inner border of its annulus, the outer border of which was $3^{\circ}$ in diameter. One display type, the luminance profile of which is illustrated in Figure $2 \mathrm{~A}$, was RCFG with the disk luminance set at $4 \mathrm{~cd} / \mathrm{m}^{2}$ for each eye, whereas the annuli were $4.5 \mathrm{~cd} / \mathrm{m}^{2}$ for one eye and $3.5 \mathrm{~cd} / \mathrm{m}^{2}$ for the other. Since the disk-to-annulus contrasts were low (about $6 \%$ and $7 \%$, respectively), the apparent lightness of the disks in this display was not dramatically different, but the disk-annulus border contrast was reversed. The other display, whose luminance profile is shown in Figure 2B, consisted of a disk of $4 \mathrm{~cd} / \mathrm{m}^{2}$ surrounded by an annulus of $36 \mathrm{~cd} / \mathrm{m}^{2}$ for one eye paired with a disk of $6 \mathrm{~cd} / \mathrm{m}^{2}$ surrounded by an annulus of $9 \mathrm{~cd} / \mathrm{m}^{2}$ in the other. In this display, the disk-annulus border contrast was not reversed eye-to-eye, but the apparent lightness of the disks was substantially different; one disk appeared darker than the other because of both a real difference in physical intensity and the contribution of simultaneous contrast.

With the RCFG stimulus, all observers experienced rivalry and none reported any difficulty in tracking phenomenal oscillations in dominance. During 1-min inspection periods, the typical observer reported seeing one or the other of the disks exclusively over $80 \%$ of the time, with the rest of the viewing period consisting of mixed states. Individual dominance durations were, on average, about $2.5 \mathrm{sec}$. When shown the other display, where diskto-annulus contrast was in the same direction in both eyes, most observers reported rivalry (i.e., perception was unstable), but all complained that for substantial periods of time the binocular disk appeared stable and intermediate in lightness relative to its monocular components. In fact, none of these observers felt that they could signal with certainty the exclusive perception of either of the monocular disks.

We conclude that the reversed contrast of the contours defining each eye's central disk is crucial for achieving compelling and unambiguous rivalry. When the polarity of contrast is identical in each eye, the monocular disk borders apparently provide strong input for a binocular fusion mechanism that can dominate perception despite binocular differences in both disk luminance and apparent lightness. In the RCFG target, the reversal of polar-
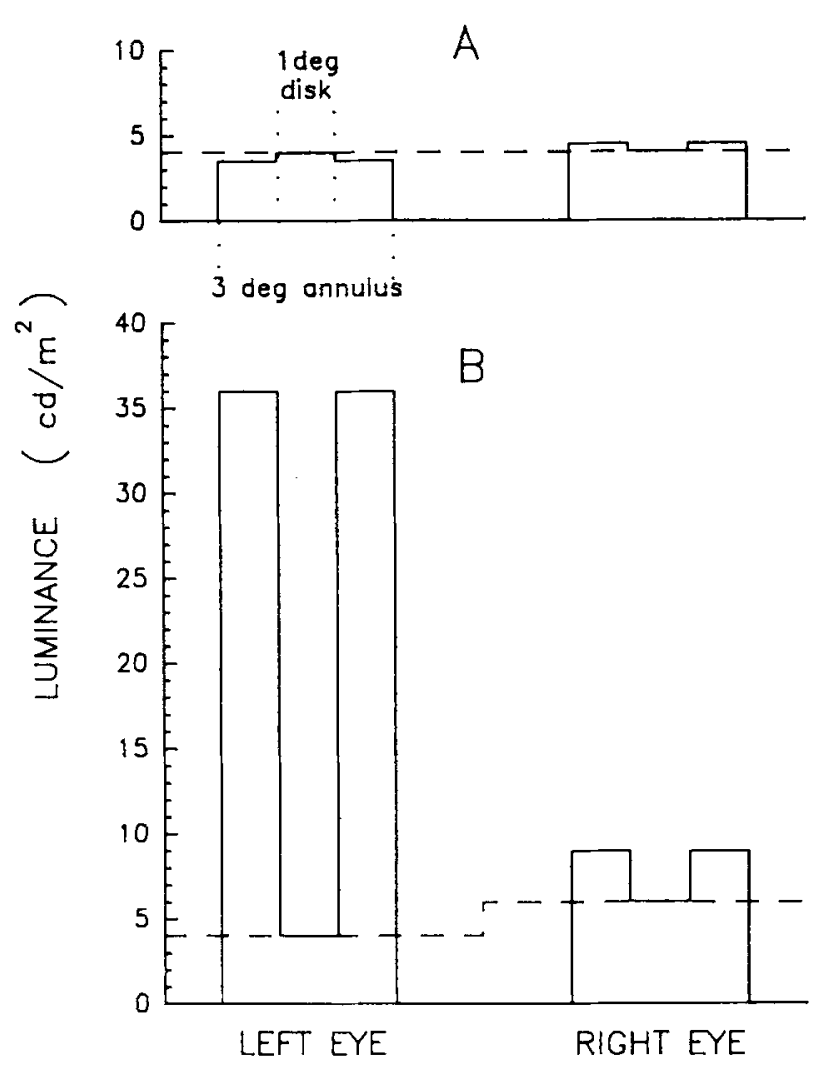

Figure 2. Luminance profiles for disk-annulus displays with (A) reversed contrast polarity and (B) identical contrast polarity in each eye. 
ity in disk-to-annulus contrast provides strong grounds for rivalry, which dominates perception despite identical disk luminance and modest differences in apparent disk lightness. In either display type, the outer border of the annulus appears fused and stable since the contrast of that border is $100 \%$ and of agreeable polarity in both eyes.

In test probe experiments, subjects are usually required to indicate (generally by manipulating a switch) when one stimulus is exclusively visible while the other is completely suppressed. During these occasions, a probe stimulus is superimposed either upon the dominating or suppressed rival target. The success of such experiments requires that the duration of these exclusive dominance episodes be sufficiently long that a probe presentation can be accomplished before the episode ends. Furthermore, in the event that the sensitivity of both eyes is being gauged within the same session, it is desirable that dominance durations for each eye be at least roughly equal. A major advantage of the RCFG rival display is that the experimenter can exert considerable control over the temporal characteristics of rivalry dominance without manipulating the rival region itself, which is necessary with more conventional targets.

It is well documented that the temporal course of rivalry alternations is influenced by the strength of the competing monocular stimuli (Levelt, 1968). With the RCFG display, stimulus strength is a function of both the disk-to-annulus contrast and the luminance of the disk. The predominance of an RCFG stimulus (i.e., the proportion of the time that the stimulus is seen to the exclusion of its rival) increases with increases in contrast, and this effect is maximal when luminance is low. Predominance also increases with increases in disk luminance, especially when disk-to-annulus contrast is low. The contrast effect is especially interesting since, with disks that are brighter than their annuli, contrast can be increased by decreasing annulus luminance. Thus an eye's predominance can be increased by removing energy from its stimulus. Of course, the effect of decreasing annulus luminance is an apparent increase in the lightness of the disk. But it is not simply apparent disk lightness that causes the disk to predominate, since we observe the same contrast effect when the luminance of an annulus surrounding a dimmer disk is increased.

Contrast and luminance also affect the mean duration of individual monocular dominance episodes. The major effect of increasing either luminance or contrast of a rival target in one eye is to shorten suppression durations in that eye, thus decreasing dominance durations of its competing target (in the other eye). This effect is expected from prior reports (e.g., Blake, 1977; Levelt, 1968). Besides abbreviating dominance durations in the competing rival target, the target whose contrast is increased also tends to dominate for longer durations, an effect consistent with recent findings reported by Mueller and Blake (1989). We have not observed this effect in response to the luminance manipulation.

To summarize, both rivalry predominance and dominance durations can be controlled in an RCFG display without modifying the rivaling disks. Dominance durations can be equalized in the two eyes by manipulating the contrast in one eye relative to that in the other. Dominance durations in an eye may be lengthened by decreasing contrast in the partner eye. Using RCFG stimuli of low contrast in both eyes favors long dominance durations and slow alternation rates.

Although in many instances slow rivalry alternations may be desirable, low contrast also tends to increase the time spent in a state of mixed dominance, where all or part of both eyes' rival targets are seen simultaneously (Hollins, 1980; Liu, Tyler, \& Schor, 1992; Liu, Tyler, Schor, \& Lunn, 1990). We find the same effect with the RCFG display. With $1^{\circ}$ disks and 1 -min inspection periods, incomplete suppression is experienced for about $13 \%$ of the period at $60 \%$ contrast and increases to about $20 \%$ at $14 \%$ contrast. We have not found, however, the proportion of the time spent in such mixed states to be appreciably influenced by variation in disk luminance (over the range from 2 to $8 \mathrm{~cd} / \mathrm{m}^{2}$ ). For comparison, we measured rivalry alternations with orthogonally oriented 6-cpd gratings of $60 \%$ contrast subtending $1^{\circ}$ in a dark field. Tracking data indicated that periods of mixed states with rivaling gratings were experienced about $67 \%$ of the time. Interestingly, when an annulus was placed around one of the rival gratings, the luminance of which was more intense than the mean luminance of the grating $(50 \%$ contrast), the proportion of the time spent in mixed states decreased to $36 \%$. Thus, the occurrence of mixed states with rivalrous gratings can be lessened by introducing reverse contrast at their outer border.

The size of rival targets also affects the occurrence of mixed states. Small target size favors unitary suppression, whereas large targets tend to produce piecemeal rivalry (Blake, O'Shea, \& Mueller, 1992). To determine the effect of target size on rivalry, we shortened the viewing distance of the previously described display so that the disks subtended $1.5^{\circ}$. When we tracked the rivalry of a pair of $50 \%$-contrast orthogonally oriented gratings $(3.3 \mathrm{cpd})$, about $70 \%$ of the viewing period was spent in mixed states. When the grating contrast was reduced to $10 \%$, mixed states increased to about $80 \%$. On the other hand, when RCFG targets with $1.5^{\circ}$ disks were viewed over the same range of contrasts, periods of mixed states never exceeded $20 \%$ of the viewing period.

When used in test probe experiments, a particularly nice feature of the RCFG stimulus is that it lends itself to a session protocol in which both eyes can be tested in a single series of test trials. When test trials that assess the sensitivity of each eye in each rival state (dominant and suppressed) are randomly intermixed, subjects are unable to anticipate the condition being tested in a trial and, therefore, a potential source of bias is reduced. For example, in our laboratory we recently used the RCFG rival target in combination with an adaptive psychometric procedure, QUEST (Watson \& Pelli, 1983), in a study of the effect of rivalry suppression on contrast sensitivity. A session in this experiment consisted of a 32-trial QUEST series for each of four conditions (each eye in each rival state). Trials in the four QUEST series were randomly interleaved. Although they involved a total of 128 trials, each session was 
typically accomplished in less than $1 / 2$ hour and yielded satisfyingly stable estimates of thresholds in each of the four conditions. We tested 3 experienced and 6 naive observers, and all showed robust threshold elevation (on the order of $1 / 2 \log$ unit) during rivalry suppression.

To summarize, the RCFG stimulus offers a number of advantages when used in studies of binocular rivalry. Predominance can be readily controlled. Individual dominance durations are relatively long, and periods of mixed states are relatively rare. Since the two eyes are equivalently stimulated in the rivalrous region and that region contains no contours, test trials for each eye in each rival state may be interleaved within a single session. Finally, we found robust suppression effects with test probes superimposed on rivaling RCFG targets.

\section{REFERENCES}

Blake, R. (1977). Threshold conditions for binocular rivalry. Journal of Experimental Psychology: Human Perception \& Performance, 3, 251-257.

Blake, R., O'Shea, R. P., \& Mueller, T. J. (1992). Spatial zones of binocular rivalry in central and peripheral vision. Visual Neuroscience, 8, 469-478.
Hollins, M. (1980). The effect of contrast on the completeness of binocular rivalry suppression. Perception \& Psychophysics, 27. 550-556.

Levelt, W. J. M. (1968). On binocular rivalry. Soesterberg, The Netherlands: Institute for Perception RVO-TNO.

LiU, L., Tyler, C. W., \& Schor, C. (1992). Failure of rivalry at low contrast: Evidence of a suprathreshold binocular summation process. Vision Research, 32, 1471-1479.

Liu, L., Tyler, C. W., SChor, C., \& LunN, R. (1990). Dichoptic plaids: No rivalry for lower contrast orthogonal gratings. Investigative Ophthalmology \& Visual Science, 31, 526.

Mueller, T. J., \& Blake, R. (1989). A fresh look at the temporal dynamics of binocular rivalry. Biological Cybernetics, 61, 223-232.

WALES, R, \& Fox, R. (1970). Increment detection thresholds during binocular rivalry suppression. Perception \& Psychophysics, 8, 90-94.

WALLACH, H., \& ADAMS, P. A. (1954). Binocular rivalry of achromatic colors. American Journal of Psychology, 67, 513-516.

Watson, A. B., \& PELlı, D. G. (1983). QUEST: A Bayesian adaptive psychometric method. Perception \& Psychophysics, 33, 113-120.

WESTENDORF, D. H. (1989). Binocular rivalry and dichoptic masking: Suppressed stimuli do not mask stimuli in a dominating eye. Journal of Experimental Psychology: Human Perception \& Performance, 15, 485-492.

(Manuscript received August 7, 1992; revision accepted for publication February 9, 1993.) 